\title{
Weekly patterns of aerosol in the United States
}

\author{
D. M. Murphy ${ }^{1}$, S. L. Capps ${ }^{1,2, *}$, J. S. Daniel ${ }^{1}$, G. J. Frost ${ }^{1,3}$, and W. H. White ${ }^{4}$ \\ ${ }^{1}$ Earth System Research Laboratory, Chemical Sciences Division, National Oceanic and Atmospheric Administration \\ Boulder, CO 80305, USA \\ ${ }^{2}$ Vanderbilt University, Nashville, TN 37240, USA \\ ${ }^{3}$ Cooperative Institute for Research in Environmental Sciences, University of Colorado Boulder, CO 80309, USA \\ ${ }^{4}$ Crocker Nuclear Laboratory, University of California, Davis Davis, CA 95616, USA \\ * now at: School of Chemical and Biomolecular Engineering, Georgia Institute of Technology, Atlanta, GA 30332, USA
}

Received: 4 December 2007 - Published in Atmos. Chem. Phys. Discuss.: 10 January 2008

Revised: 13 March 2008 - Accepted: 3 April 2008 - Published: 22 May 2008

\begin{abstract}
Data from the Interagency Monitoring of Protected Visual Environments (IMPROVE) network of aerosol samplers and NOAA monitoring sites are examined for weekly cycles. At remote and rural sites, fine particle elemental carbon, crustal elements, and coarse particle mass had pronounced (up to $20 \%$ ) weekly cycles with minima on Sunday or Monday. Fine particle organic carbon and mass had smaller amplitude cycles, also with Sunday or Monday minima. There was no statistically significant weekly cycle in fine particle sulfate despite a 5 to $15 \%$ weekly cycle in power plant $\mathrm{SO}_{2}$ emissions. Although results for nitrate may be more susceptible to sampling artifacts, nitrate also showed a pronounced weekly cycle with an amplitude similar to elemental carbon. The only species found with a weekend maximum was $\mathrm{Pb}$, probably from general aviation on weekends. Aerosol optical properties at NOAA monitoring sites were consistent with the IMPROVE chemical data, with significant weekly cycles in aerosol light absorption but not light scattering. These results support a large role of diesel emissions in elemental carbon aerosol over the entire United States and suggest that a large fraction of the airborne soil dust is anthropogenic. They also suggest that studies of weekly cycles in temperature, cloudiness, precipitation, or other meteorological variables should look for causes more in light-absorbing particles and possible ice nucleation by dust rather than sulfate or total aerosol. There are also implications for personal exposure and epidemiological studies of aerosol health effects.
\end{abstract}

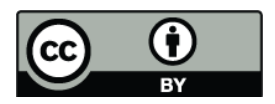

Correspondence to: D. Murphy (daniel.m.murphy@noaa.gov)

\section{Introduction}

Observations of the weekly cycle are a powerful tool for distinguishing anthropogenic from natural causes. Only anthropogenic activities are likely to influence concentrations, temperatures, or other atmospheric variables on a seven-day cycle. Weekly trends have been found for diurnal temperature range over the United States (Forster and Solomon, 2003) and China (Gong et al., 2006) as well as cloudiness and precipitation in Germany (Bäumer and Vogel, 2007). White et al. $(1990,1991)$ studied transport of urban pollutants using weekly cycles. Blanchard and Tanenbaum (2003) found significantly less $\mathrm{NO}_{2}$ on weekends in Southern California. Beirle et al. (2003) found weekly cycles in column $\mathrm{NO}_{2}$ measured from satellite, with different amplitudes and phases on various continents that could be related to various societies. A number of studies have examined weekly cycles of ozone concentrations and photochemistry (e.g. Elkus and Wilson, 1977; Marr and Harley, 2002; Murphy et al., 2007).

Several studies have specifically studied weekly cycles of aerosol. Almeida et al. (2006) found that chemical elements such as $\mathrm{Ca}, \mathrm{Si}$, and $\mathrm{Fe}$ associated with soil were significantly higher on weekdays at a site in a suburb of Lisbon. Motallebi et al. (2003) found weekly cycles at selected urban sites in California. Lough et al. (2006) and Harley et al. (2005) found significant weekly trends in aerosol components in the Los Angeles area. In particular, both found much lower ratios of elemental carbon to organic carbon on weekends. Jin et al. (2005) used satellite data to discern a midweek maximum in aerosol optical thickness near New York City. Bäumer et al. (2008) examined aerosol optical thickness over Europe. Delene and Ogren (2002) found slightly higher single scattering albedos on Sundays and Mondays at Bondville, Illinois

Published by Copernicus Publications on behalf of the European Geosciences Union. 


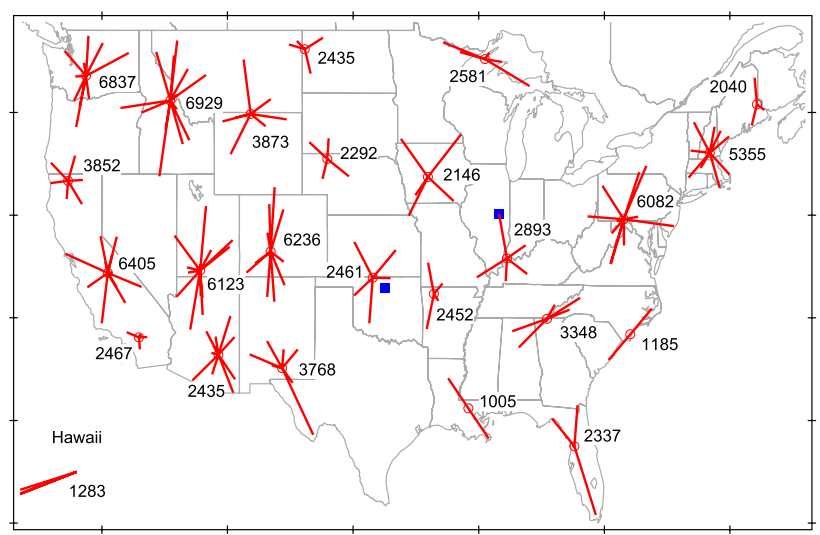

Fig. 1. Clusters generated from the IMPROVE site locations. Each cluster is shown by a circle with lines to the positions of the individual sites in that cluster. Next to each cluster is the number of elemental carbon samples for the analysis in this paper; other species have similar numbers of samples. Also shown as squares are the Bondville and Southern Great Plains NOAA monitoring sites discussed in this paper.

and Southern Great Plains, Oklahoma than on other days of the week.

With the exception of Delene and Ogren (2002), studies on weekly aerosol properties have concentrated on urban sites. In this paper we use data from the IMPROVE network and NOAA aerosol monitoring sites to examine weekly cycles in aerosol across the United States. An important feature of the IMPROVE aerosol data set is the chemical analysis, allowing much better weekly analysis than a simple mass measurement.

\section{Data}

\subsection{IMPROVE network}

IMPROVE data in this paper are from 1 September 2000 through 31 August 2006. This is an integral number of weeks and years, which helps keep annual cycles from being aliased into weekly cycles. We use data after summer 2000 because the network shifted then from a Wednesday-Saturday sampling schedule to a once every third day schedule that rotates through the days of the week, an important feature for this work. There were also few sites in the eastern United States before 2000. Except as noted for coarse mass, all data in the paper are for fine mode aerosol smaller than $2.5 \mu \mathrm{m}$.

The IMPROVE monitoring program is described by Malm et al. (2004). Routine monitoring began in 1988 with the objectives of establishing current background haze levels, identifying the chemical composition and emissions sources of regional aerosols, and documenting long-term trends in aerosol concentrations. Most of the samplers are in locations representative of the 156 National Parks and Wilderness Ar- eas where the Clean Air Act provides special protections for visibility. The network now includes about 170 rural or remote sites in the United States.

Data, standard operating procedures and site descriptions are all available in detail at http://vista.cira.colostate.edu/ improve/. Every third day (all sites sample on the same days), a set of three $\mathrm{PM}_{2.5}$ samples and one $\mathrm{PM}_{10}$ sample is collected by four independent filter sampling trains. The $\mathrm{PM}_{10}$ filter is analyzed only for mass. The $\mathrm{PM}_{2.5}$ modules sample behind AIHL cyclones (John and Reischl, 1980) at nominal flow rates of $22.81 \mathrm{~min}^{-1}$. One module collects $\mathrm{PM}_{2.5}$ on a $37 \mathrm{~mm}$ Nylon filter behind an annular denuder coated with $\mathrm{Na}_{2} \mathrm{CO}_{3}$ and glycerol. This filter is analyzed by ion chromatography for the anions chloride, nitrate, and sulfate. A second module collects undenuded $\mathrm{PM}_{2.5}$ on a $25 \mathrm{~mm}$ Teflon filter for weighing and energy-dispersive $\mathrm{x}$-ray fluorescence analysis (XRF). The third module collects undenuded $\mathrm{PM}_{2.5}$ on a $25 \mathrm{~mm}$ quartz-fiber filter for thermal fractionation of organic and elemental carbon with an optical correction for pyrolysis.

The methods used for elemental and carbon analysis have undergone some minor changes since August 2000. Before December 2001, the elements lighter than iron were determined by proton-induced x-ray emission, and since January 2005 the XRF analysis for elements lighter than nickel has been done under vacuum. Carbon analyses since January 2005 have been conducted with new instrumentation according to a more precise protocol (Chow et al., 2007). These one-time method transitions should have no effect on multiyear weekly cycles.

There is a weekly pattern of sampler operations: the local operator checks the system's operation each Tuesday, and replaces the cartridge of exposed filter cassettes in each module with one of unexposed filter cassettes. This schedule imposes extra handling for Tuesday samples, which are briefly interrupted while the operator transfers the active filter cassette from the old cartridge to the new one. It also imposes sample-day-dependent delays between exposure and unloading, when losses might potentially occur within the sampler. (Any potential for contamination would presumably exist both before and after exposure, and so be independent of sample day.) Such hypothetical effects have not been detected in previous testing. With the exception of winter nitrate, they would not explain the calendar-week cycles presented here, whose extremes usually occur away from the Tuesday sampler maintenance.

The sites in the IMPROVE database were combined into 25 groups using hierarchical cluster analysis (Fig. 1). Fifteen urban sites were eliminated (ATLA1, BALT1, CHIC1, DETR1, FRES1, HOUS1, NEYO1, OLTO1, OMAH1, PHOE1, PITT1, PUSO1, RUBI1, SAGU1, and WASH1), as were some sites that were closed before 2000. Then great circle distances were calculated between all site pairs and the closest pair of sites or clusters combined with each other. The process was repeated until the closest clusters were more 
than $390 \mathrm{~km}$ apart. This threshold was chosen to keep separate clusters in California. Single sites that had not ended up in a cluster were paired together (in Louisiana and the Carolinas) or combined with the nearest cluster (Everglades and Big Bend). Of sites outside of the continental United States, only Hawaii was kept because there were fewer samples in Alaska or the Virgin Islands.

Use of clusters is essential to this work. Weekly cycles at single sites are often not statistically significant when samples are taken every third day. Several sampling sites must be averaged to distinguish weekly cycles from random variability. Yet one cannot average the entire United States without first looking at regional patterns. It does not make sense to average together mineral dust concentrations from Arizona and Maine or sulfate concentrations in California and Ohio. The exact technique used to generate the clusters is less important than having some sort of regional averaging.

To start with a consistent set of data, all samples were required to have valid values for mass, elemental carbon, and sulfate. About $0.1 \%$ of the data were eliminated as high outliers. These are probably valid data influenced by local events such as fires or nearby dust generation. For example, fine mode mass was required to be less than $50 \mu \mathrm{g} \mathrm{m}^{3}$. The results are not sensitive to these cutoffs but they do help keep a single sample from skewing one day of the week for an entire cluster. This left 92744 samples in the IMPROVE data during the time period after eliminating the urban sites. The actual number of samples for a given species may be slightly smaller, since not all analyses were performed on every sample. Data below detection limit were retained as zeros but species with many values below detection limit are not discussed in the paper. Notably, our mineral dust analysis does not consider aluminum because of a poor detection limit compared to silicon and iron.

Most of the subsequent results are presented in terms of the average weekly cycle in a cluster. For each day of the week, this is calculated as the average of all observations on that day of the week during the September 2000 to August 2006 period, at all sites in the cluster. For visual presentation, and for network averages, each cluster's cycle is normalized to that cluster's overall September 2000 to August 2006 mean.

There is an option whether or not to normalize data from individual sites before calculating the weekly cycles for each cluster. Normalizing prevents a few sites with high absolute concentrations from dominating the averages, but has the disadvantage of amplifying noise from sites with low concentrations that may be near detection limits. Normalizing can also overemphasize sites with incomplete records. We have chosen to directly average all data within a cluster. We have done the calculations both ways and the calculated weekly cycles are not sensitive to whether or not the sites are normalized before averaging. In part, this is because the exclusion of urban sites leaves sites within each individual cluster that usually have similar absolute concentrations. For a particular species, the standard deviations of the concentrations of the sites in individual clusters are usually about half as large as the standard deviation of the clusters from the continental average.

Judging the statistical significance of the weekly cycles is difficult. The most important problem is the number of independent measurements. The standard errors in subsequent figures are calculated as usual by dividing the standard deviation of individual measurements by the square root of the number of measurements. The samples are not completely independent of each other, however, as this calculation implicitly assumes. Concentration variations are driven largely by meteorological variables, which are correlated in both time and space. The IMPROVE protocol of samples every third day should reduce but not completely eliminate the autocorrelation between successive samples (Anderson et al., 2003). Similarly, multiple sites within a cluster will often lie in the same synoptic airmass.

Dilution can cause statistical significance to be underestimated. Consider the simple case of two sites downwind of a consistent weekly source with rapid transport. Both would show the same weekly cycles, but the site further downwind would have lower absolute values. If the sites with different absolute concentrations are averaged together, the standard deviations for each day would be large even though the sites were sampling the same source.

To assess the statistical significance, we compared the amplitude of the observed weekly cycle with the amplitude of the weekly cycle for synthetic time series generated from randomized versions of the same data. To preserve shortterm correlations, data within each month were kept as a block and each month was shifted by a random number of days of the week. The resulting statistics for 500 randomized samples of the data are shown in Table 1. The weekly cycle inferred from random data varies by species because of different variance and skew in the concentrations of each species.

Since we are considering the absolute value of the weekly cycle in the randomized data, the distributions are not centered on zero. This is why the 90th and 99th percentiles may appear closer than expected. Also, these percentiles of the absolute values of weekly cycles represent the amplitude required for confidence that a cycle of any phase is real. They are not the same as the 90 percent confidence limits on the amplitude of the derived cycles (these are not computed here). The reason the two quantities are different is that random noise will always generate a positive weekly cycle of some phase whereas it can generate either a positive or negative error in a cycle whose phase is already determined.

We also checked for cycles of 5, 8, and 10 days using the same software as the 7-day cycle (6 and 9 day cycles do not work well with samples taken every third day). For the species with cycles that are statistically significant compared to the randomized data, the 7-day cycle was much larger than the other periods. For example, by one measure (maximum two consecutive days minus minimum two consecutive days) 
Table 1. Statistics of weekly cycles for selected species averaged over the continental United States.

\begin{tabular}{llcccc}
\hline Species & $\begin{array}{l}\text { Overall continental } \\
\text { US average }\end{array}$ & $\begin{array}{c}\text { Weekly range } \\
(\%)\end{array}$ & $\begin{array}{c}\text { Weekly two-day } \\
\text { range* }(\%)\end{array}$ & $\begin{array}{c}\text { Randomized data weekly two-day range } \\
(\%)\end{array}$ \\
\hline & $\left(\mu \mathrm{g} \mathrm{m}^{-3}\right)$ & & & 90 th percentile & 99 th percentile \\
Silicon & 0.149 & 22.4 & $\mathbf{1 5 . 3}$ & 9.1 & 11.4 \\
Iron & 0.040 & 26.5 & $\mathbf{1 8 . 1}$ & 10.2 & 14.2 \\
Calcium & 0.046 & 24.3 & $\mathbf{1 7 . 4}$ & 9.6 & 12.8 \\
Coarse mass & 4.91 & 23.1 & $\mathbf{1 8 . 4}$ & 9.8 & 12.8 \\
Elemental carbon & 0.230 & 14.2 & $\mathbf{1 1 . 4}$ & 6.3 & 6.1 \\
Organic carbon & 1.159 & 7.9 & 5.6 & 4.9 & 6.4 \\
Fine mass - EC & 5.90 & 7.9 & 6.2 & 5.1 & 7.2 \\
Fine mass - (EC+soil) & 5.17 & 5.5 & 4.8 & 5.4 & 7.2 \\
Sulfate & 1.835 & 4.5 & 2.3 & 5.4 & 6.7 \\
Nitrate & 0.592 & 13.4 & $\mathbf{1 1 . 0}$ & 8.0 & 5.2 \\
$\mathrm{~Pb}$ & 0.0012 & 9.1 & $\mathbf{6 . 7}$ & 5.0 & 5.3 \\
$\mathrm{~Pb} /(\mathrm{Pb}+\mathrm{Zn})$ & 0.26 (ratio) & 8.4 & $\mathbf{7 . 0}$ & 3.6 & 8.0 \\
$\mathrm{Zn}$ & 0.0041 & 8.3 & $\mathbf{6 . 6}$ & 4.3 & 5.9 \\
$\mathrm{~V}$ & 0.0008 & 4.5 & 3.2 & 6.6 & \\
$\mathrm{Se}$ & 0.0005 & 3.3 & 1.8 & 4.4 & \\
\hline
\end{tabular}

* maximum two consecutive days - minimum two consecutive days.

Bold: exceeds 99th percentile of randomized data; italics: less than 90th percentile.

the weekly cycle for silicon was $15.3 \%$. The 5,8 , and 10 day cycles were $2.6 \%, 2.7 \%$, and $5.6 \%$ respectively. For sulfate, which was not statistically significant, the weekly cycle was $2.3 \%$ and the 8 -day cycle was $2.5 \%$. Finally, all of the weekly patterns shown here are robust with respect to changing the time period to various subsets of 2000 to 2006 .

\subsection{NOAA aerosol optical properties}

The National Oceanic and Atmospheric Administration (NOAA) continuously measures aerosol optical properties at a number of baseline and regional monitoring stations (Delene and Ogren, 2002). Bondville, Illinois, and Southern Great Plains, Oklahoma, are relevant to this work. Their locations are shown on Fig. 1. Daily averaged data were used to construct the weekly cycles.

Aerosol light absorption was measured at $565 \mathrm{~nm}$ using Particle Soot/Absorption Photometers (PSAP, Radiance Research). The data were corrected for scattering using Bond et al. (1999). Aerosol light scattering was measured using TSI 3563 nephelometers. Only the $550 \mathrm{~nm}$ data are shown here. The sample stream was heated, if necessary, to maintain the relative humidity at about $50 \%$. Further descriptions are available at http://www.esrl.noaa.gov/gmd/ aero/instrumentation/instrum.html.

The PSAP filters were changed less frequently on weekends at the Southern Great Plains sit and therefore had lower average total filter transmission on Sundays and Mondays (J. Ogren, personal communication, 2007). The Bondville site was less affected. More recent work on PSAP corrections by Virkkula et al. (2005) suggests that the Bond et al. cor- rections have an accurate dependence on filter transmission except for highly absorbing aerosols (albedo less than about 0.7 ) that are not likely to be important for these sites.

\subsection{Emissions inventories}

Weekly cycles in $\mathrm{SO}_{2}$ and $\mathrm{NO}_{\mathrm{x}}$ emissions from power plants were computed for comparison with observed weekly cycles in aerosols. US power plant emission data were obtained from the Environmental Protection Agency's Clean Air Markets Division, http://camddataandmaps.epa.gov/gdm/index. cfm?fuseaction=emissions. wizard. 1999 and 2005 hourly emissions recorded by continuous emission monitors at each power plant were aggregated over large geographic regions containing 50 to 300 individual plants. The hourly emissions for each region were averaged for each day of week over the entire year, and the day-of-week averages were normalized using the average emissions in each region. Weekly cycles in 1999 were very similar to the 2005 data shown in Fig. 6 .

\section{Results}

\subsection{Crustal elements}

The largest percentage weekly cycles in aerosol concentrations were observed for the crustal elements $\mathrm{Si}, \mathrm{Ca}, \mathrm{Ti}$, and Fe (Fig. 2). All had weekly cycles with $20 \%$ or greater range. Minima were on Sunday for most locations and maxima were usually on Thursday. Aluminum also had similar patterns but more values below the detection limit. The weekly cy- 

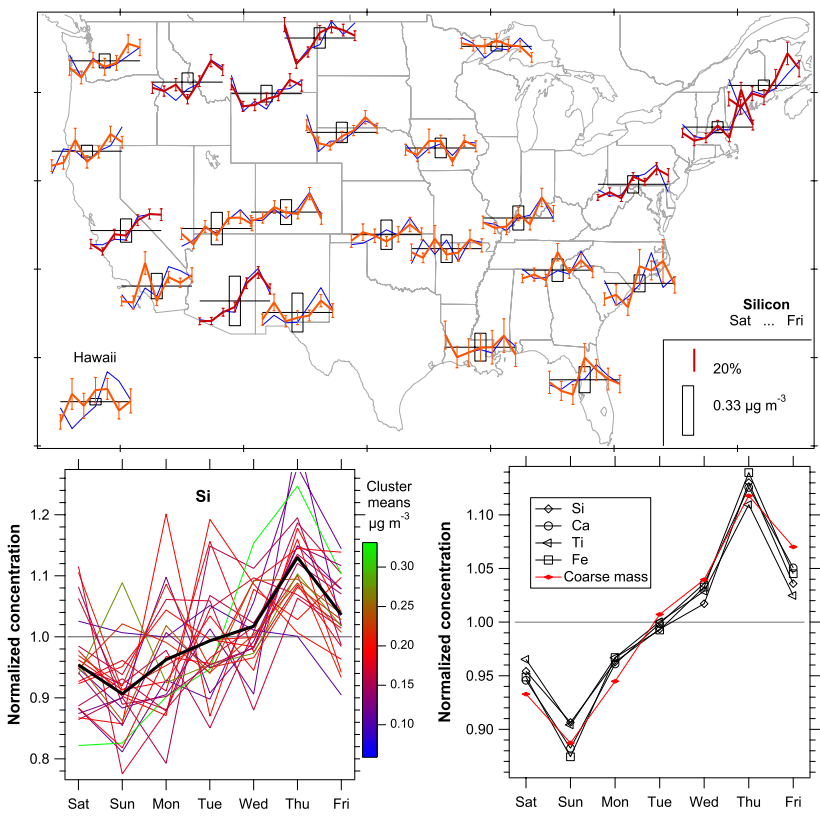

Fig. 2. Weekly cycles of crustal elements in the fine mode (below $2.5 \mu \mathrm{m})$. The top panel shows weekly cycles for silicon at each cluster of IMPROVE sites. For each cluster of sites, Saturday through Friday averages are shown in red or orange as departures from the weekly mean, with standard errors. Red curves indicate statistical significance is more likely than for orange. Weekly cycles in medians are shown in blue. Rectangles are proportional in size to the annual mean for each cluster. The lower left panel shows the normalized weekly cycles for silicon for each cluster in the continental United States. The heavy line is the average over all clusters. The lower right panel shows the average weekly cycle for the continental United States for various crustal elements and coarse mass, defined as the difference between fine and total $\left(\mathrm{PM}_{10}\right)$ mass.

cles were consistent throughout the country except for a few locations where the absolute values were small. One of the largest weekly cycles was observed for the cluster in Arizona that had the largest annual averages for these elements of any cluster in the continental United States. That cluster had low values on both Saturday and Sunday, perhaps because the soil particles were generated locally so the atmospheric concentrations could respond more quickly to low emissions on the weekend. A curious feature for many locations is that the weekly maximum was on Thursday rather than Friday, as might be expected if weekday emissions built up in the atmosphere throughout the week. It is not clear to what extent this is a statistical fluctuation or a consequence of lower emissions on Friday caused by four-day work weeks.

On Fig. 2 and subsequent figures, the average for the continental United States was generated from the normalized averages of the clusters. This means that individual sites in areas with a dense network of sites contribute less to the average than sites where the network is sparse. Averaging the normalized weekly cycles means that regions with low absolute

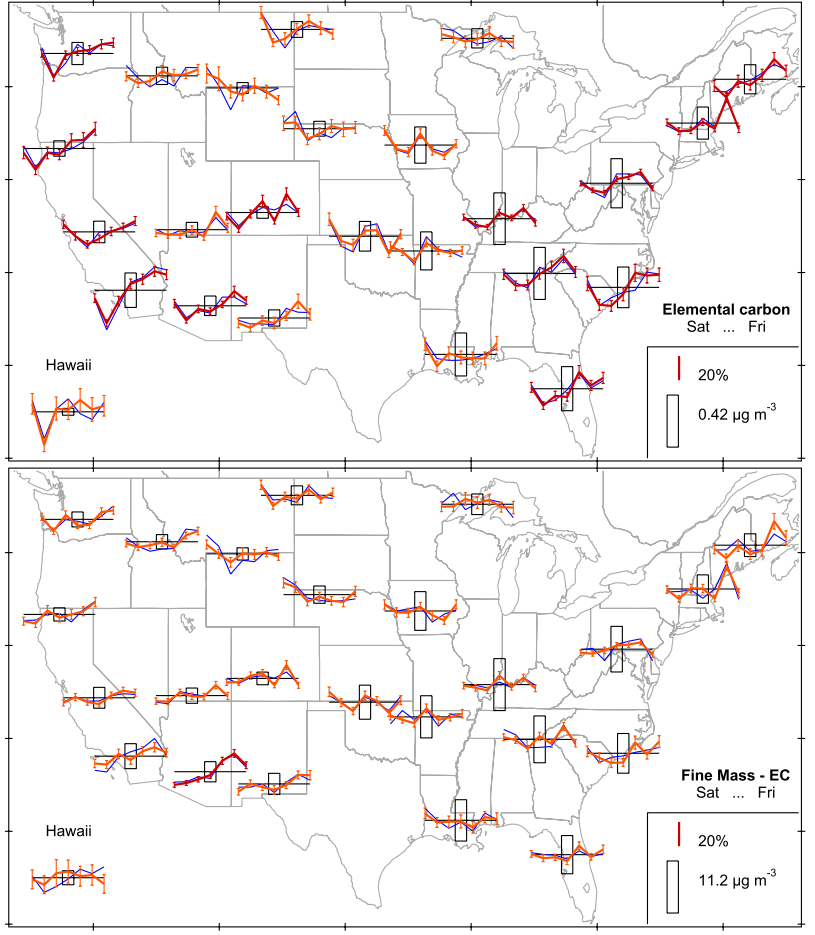

Fig. 3. Weekly cycles for fine mode elemental carbon and the balance of fine mass. As in Fig. 2, red lines are average percentage daily departures from the mean for that cluster and blue lines are departures of the medians. Rectangles are proportional to the annual mean for each cluster of sites.

concentrations contribute as much to the continental average as regions with high concentrations. As a guide to the eye, normalized weekly cycles for individual clusters are plotted in orange or red if the maximum of two consecutive days is less or more than four standard errors from the minimum of two consecutive days.

\subsection{Elemental carbon, organic carbon, and aerosol mass}

Weekly cycles for elemental carbon, organic carbon, and several measures of aerosol mass are shown in Figs. 3 and 4. Elemental carbon is subtracted from fine mode mass because for climate forcing calculations it is sensible to separate absorbing and non-absorbing aerosols. This also facilitates comparison with the optical measurements discussed later. The weekly cycles for fine mass are not statistically significant for individual clusters. Although Fig. 3 shows one highly significant cluster, testing with the randomized series shows that by chance one out of 25 clusters can exceed the four standard error threshold for the color code on Fig. 3. However, the continental US average weekly cycle for fine mass is statistically significant (Table 1) at about the $95 \%$ level. 

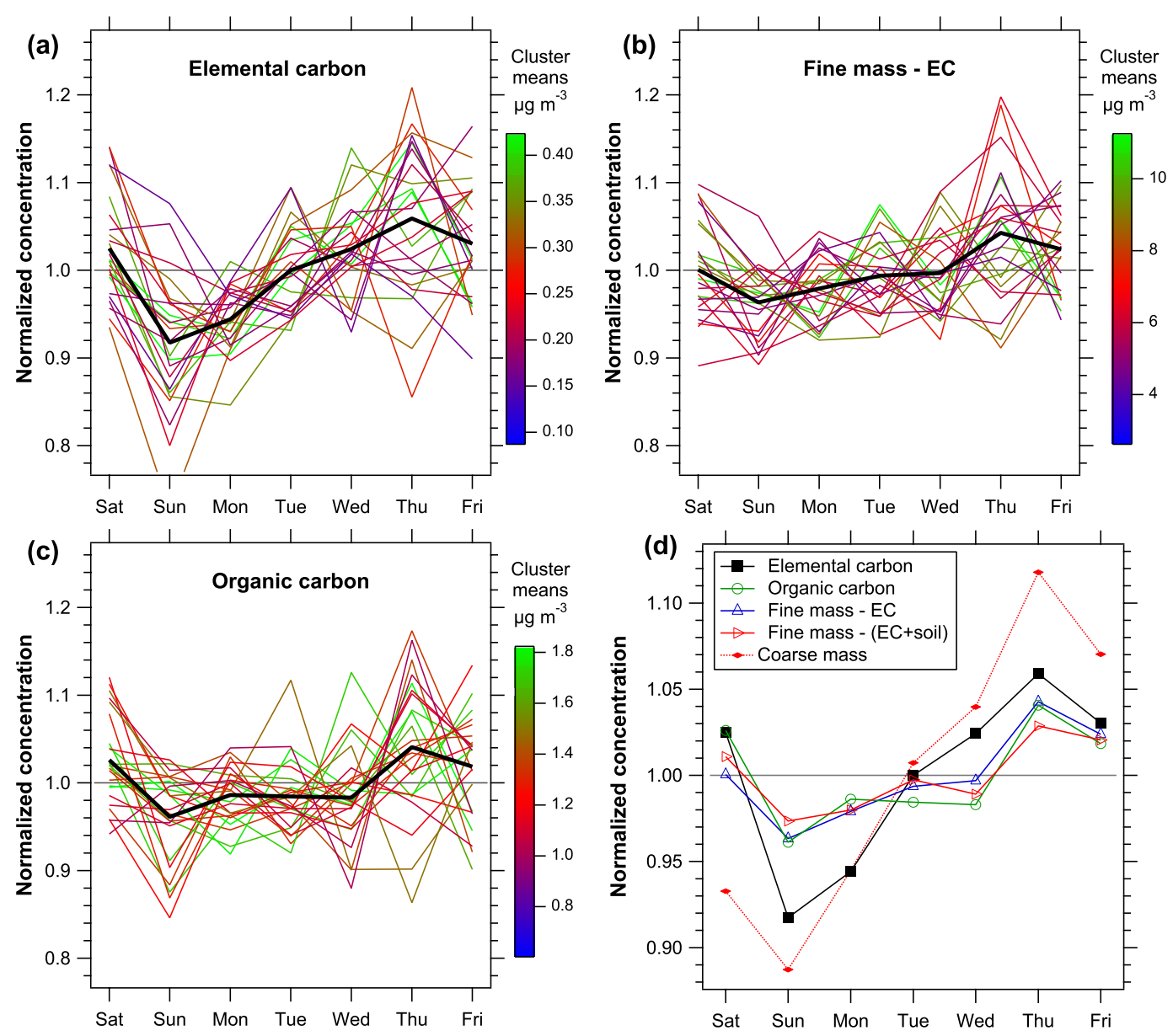

Fig. 4. A comparison of the weekly cycles of elemental and organic carbon as well as various measures of the fine and coarse mode mass. Each line is the weekly cycle for one cluster of sites, colored by the annual mean. The heavy black lines are the averages for all clusters in the continental United States.

Elemental carbon has a much larger weekly cycle than organic carbon or the fine mode mass. Figure $4 \mathrm{~d}$ compares some of the weekly cycles. In view of the large weekly cycle for crustal elements, a curve is shown for fine mass after removing the contribution from soil calculated using an equation modified slightly from Malm and Hand (2007):

Soil $=(2.42+1.5) \cdot \mathrm{Fe}+1.63 \cdot \mathrm{Ca}+2.2 \cdot \mathrm{Al}+2.49 \cdot \mathrm{Si}+1.94 \cdot \mathrm{Ti}$

The factors account for oxides, and in the case of Fe, crustal elements such as $\mathrm{K}$ that cannot easily be separated from other sources. The additional factor of $1.5^{*} \mathrm{Fe}$ is a very rough estimate of a contribution from total carbon in the soil estimated from examining some strong dust events. We infer from this curve that the weekly cycle for fine mass is only partially due to the strong cycle in soil dust. It is also due to organic carbon and nitrate.

Figure 5 shows histograms of elemental carbon concentrations for one cluster. The lower averages on Sunday and Monday were caused by a shift of the entire distribution rather than changes in a few extreme values. This is the case for other clusters as well. The histograms also show the form of the distributions: on a log scale such as Fig. 5 the distributions are skewed left. On a linear scale they are skewed to the right, so the distributions are between log-normal and normal.

The IMPROVE network uses a thermo-optical method to separate organic and elemental carbon. For all thermooptical methods, there are questions about the absolute accuracy of how organic carbon that chars during heating is separated from elemental carbon (Novakov et al., 2005; Chow et al., 2007). Despite these questions, weekly cycles depend more on consistency of the carbon analysis than the absolute cut point between elemental and organic carbon. In addition, the different weekly cycles for elemental and organic carbon show that the operational definition of elemental and organic carbon in the IMPROVE protocol does represent a useful separation for species with differing sources and behavior in the atmosphere. 


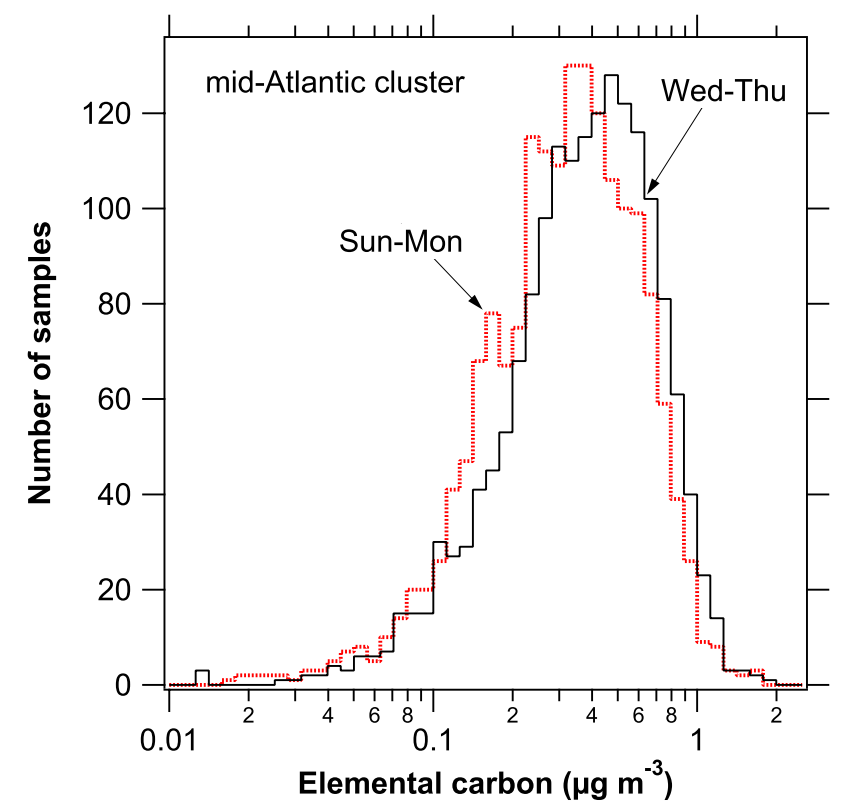

Fig. 5. Histograms of elemental carbon concentrations for one cluster of IMPROVE sites centered around Pennsylvania and West Virginia. The histograms compare Sunday and Monday with Wednesday and Thursday.

\subsection{Sulfate and nitrate}

Sulfate has a very different pattern than elemental carbon. There is no statistically significant weekly cycle in sulfate, despite a weekly cycle in $\mathrm{SO}_{2}$ emissions (Fig. 6). This is probably caused both by the emissions and the chemistry of $\mathrm{SO}_{2}$. Emissions of $\mathrm{SO}_{2}$ from power plants decline by 5 to $15 \%$ on weekends compared to weekdays, a much smaller change than the approximately $75 \%$ reduction in diesel emissions on weekends in California (Chinkin et al., 2003; Motallebi et al., 2003). Electric power generation accounted for about $70 \%$ of US $\mathrm{SO}_{2}$ emissions during the period 2000-2006 (National Emissions Inventory (NEI) Air Pollutant Emissions Trends Data, http://www.epa.gov/ ttn/chief/trends/index.html). Unlike crustal material or elemental carbon, the $\mathrm{SO}_{2}$ emissions must be chemically transformed to produce sulfate. This will delay and spread out any weekly pattern. Also, oxidation of $\mathrm{SO}_{2}$ to sulfate takes place by both gas phase reactions and liquid phase chemistry in clouds. These pathways proceed at different rates, further spreading out any weekly pattern. The variable presence of clouds may add to the daily scatter in sulfate and make cycles harder to discern.

We compared the weekly cycles for winter and summer sulfate concentrations. Sulfate concentrations are generally higher in summer than in winter, when photochemistry is slower and liquid phase clouds are less common in the northern United States. Distinguishing seasons did not reveal any statistically significant cycles.
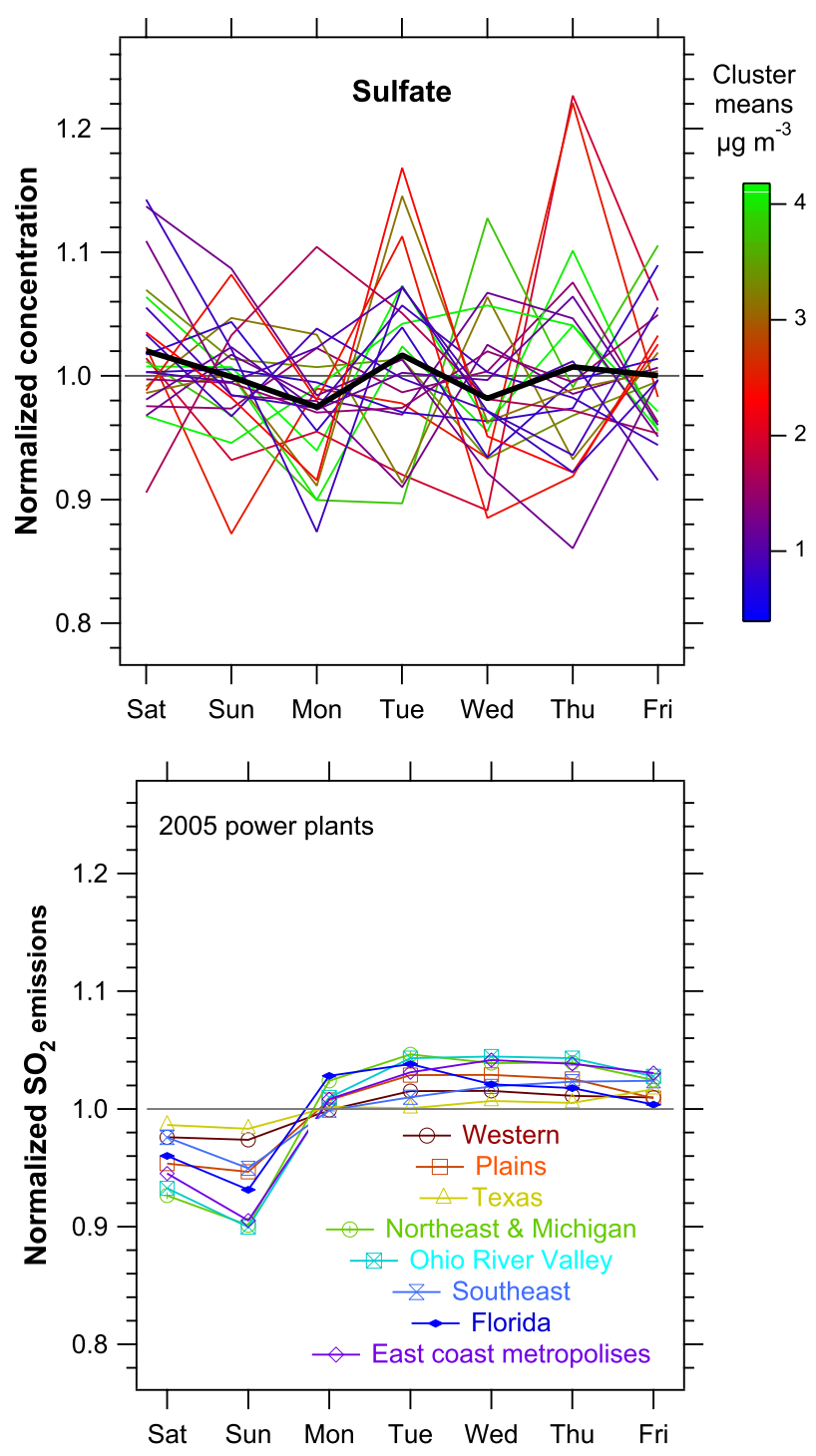

Fig. 6. Weekly cycles of sulfate in the IMPROVE network and emissions of $\mathrm{SO}_{2}$ from power plants. Symbols and lines are the same as Fig. 2.

There are two main types of aerosol nitrate: ammonium nitrate and nitrate chemically bound to crustal cations such as calcium (Murphy et al., 2006). With few exceptions, ammonium nitrate is only stable when the sulfate has been completely neutralized. Otherwise it dissociates to gas phase ammonia and nitric acid (Bassett and Seinfeld, 1984). Because of the widespread oxidation of $\mathrm{SO}_{2}$ to sulfuric acid, ammonium nitrate is more often limited by ammonium than the availability of gas phase nitric acid. The absolute amounts of nitrate vary greatly, with much larger concentrations in southern California and the Midwest than the mountain west. California and the Midwest are locations with substantial sources of ammonia and, especially for California, small emissions of $\mathrm{SO}_{2}$. The reaction of nitric acid with calcium in mineral dust is much less sensitive to sulfate. 

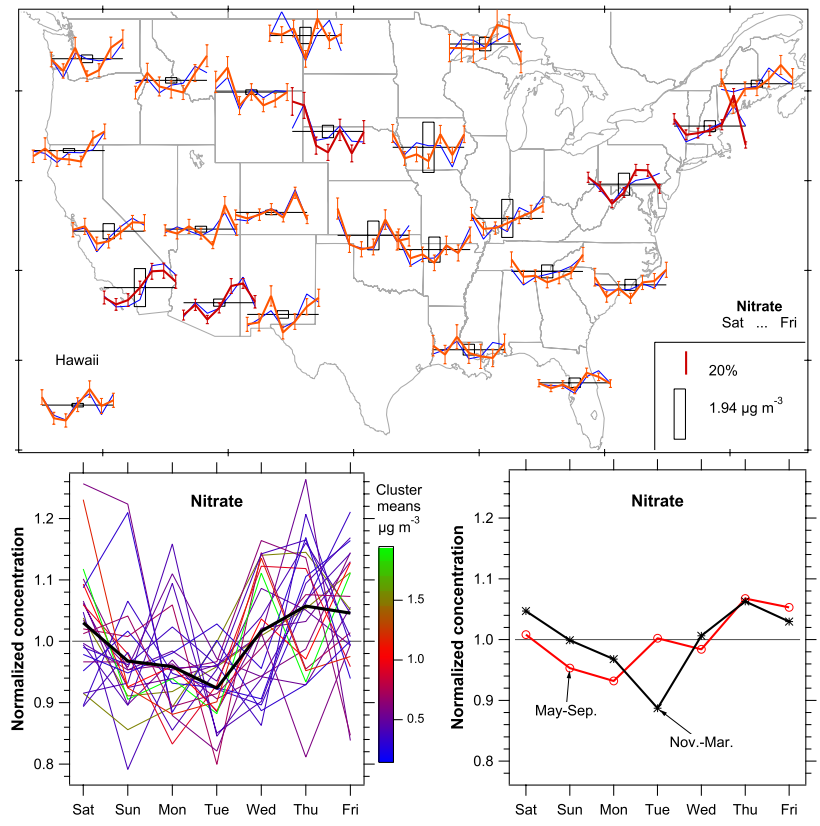

Fig. 7. Weekly cycles of nitrate in the IMPROVE network. The lower right panel shows the average for the continental US separated by season. Symbols and lines are the same as Fig. 2.

In contrast to sulfate, nitrate shows a weekly cycle (Fig. 7). Some of the cycle in nitrate is driven by the strong weekly cycle in crustal elements, but examination of the absolute values shows that calcium nitrate and similar species cannot account for all of the observed cycle in nitrate. The weekly nitrate minimum is Sunday through Tuesday, later than other species. The presence of a weekly cycle in nitrate is a little surprising because of the possible limitations by ammonia. For example, emissions of ammonia from farm animals should be fairly constant. $\mathrm{NO}_{\mathrm{x}}$ emissions from power plants have a similar cycle to that shown for sulfate in Fig. 6 . $\mathrm{NO}_{\mathrm{x}}$ emissions from vehicles have a significant component from heavy vehicles, so vehicular emissions will have a much stronger weekly cycle than $\mathrm{NO}_{\mathrm{x}}$ from power plants (Chinkin et al., 2003). The fraction of US $\mathrm{NO}_{\mathrm{x}}$ emitted by electric power generation decreased from $24 \%$ in 2000 to $20 \%$ in 2006. During the same period, on-road mobile sources produced about $37 \%$ while the contribution of off-highway vehicles has increased from $18 \%$ to $22 \%$ (National Emissions Inventory (NEI) Air Pollutant Emissions Trends Data, http://www.epa.gov/ttn/chief/trends/index.html). A weekly cycle for nitrate with a Sunday-Monday minimum has also been found in some urban areas (Millstein et al., 2007).

Although the data are noisy when separated by season, there does appear to be a shift in the timing from a Monday minimum in summer to a Tuesday minimum in winter (Fig. 7). There are two possible explanations for this shift. First, conversion of $\mathrm{NO}_{\mathrm{x}}$ to $\mathrm{HNO}_{3}$, followed by rapid equilibration with aerosol nitrate, will be much slower in winter
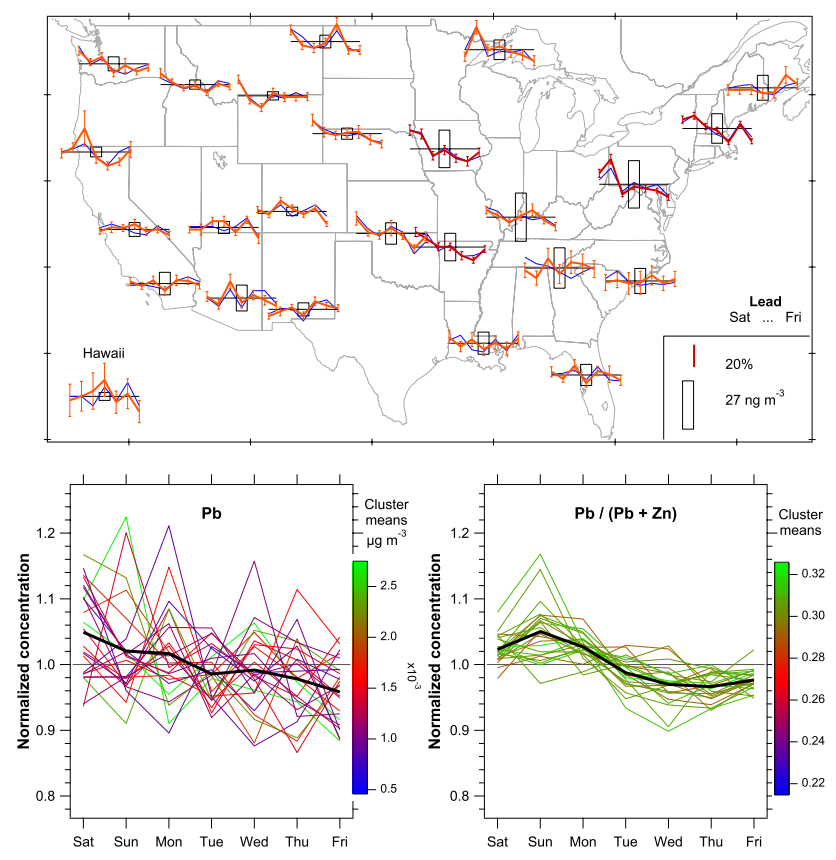

Fig. 8. Weekly cycles for $\mathrm{Pb}$ and the ratio of $\mathrm{Pb}$ to $\mathrm{Pb}+\mathrm{Zn}$. As for other figures, the red lines are the departures of the daily averages from each cluster mean, blue departures for medians, and the boxes are proportional to the annual mean for each cluster.

than in summer. That could shift the phase of the weekly cycle. Second, there could be an unrecognized artifact associated with the extra handling Tuesday samples receive from the site operator. Low temperatures and low sulfate concentrations favor the stability of ammonium nitrate particles, so nitrate concentrations in most of the country peak during the winter. The Nylon filters used for IMPROVE's nitrate measurement retain any nitric acid gas released after collection by thermal dissociation of ammonium nitrate. Warming of winter-Tuesday samples during the brief handling period, when any volatilized nitric acid would not be drawn back into the filter, must for the moment be considered as a possible explanation for the nitrate minimum on winter Tuesdays.

\subsection{Lead and other metals}

Lead is the only species studied with a weekend maximum (Fig. 8). The largest single source of airborne $\mathrm{Pb}$, accounting for about $25 \%$ of US emissions, is leaded gasoline in small piston aircraft (Murphy et al., 2007). Many of these small planes are recreational and emissions probably peak on weekends. The next largest sources of airborne $\mathrm{Pb}$ are coalfired power plants and heavy steel industry. Those emissions probably go down slightly on weekends as shown earlier for $\mathrm{SO}_{2}$.

Zinc and $\mathrm{Pb}$ are highly correlated in atmospheric samples, probably because of similar electric utility and industrial sources and similar condensation in combustion exhaust 
(Murphy et al., 2007). Their ratio is less variable than their absolute concentrations with a very consistent Sunday maximum (Fig. 8). Most locations in the United States have $\mathrm{Pb} /(\mathrm{Pb}+\mathrm{Zn})$ in a narrow range between 0.3 and 0.33 . The ratio $\mathrm{Pb} /(\mathrm{Pb}+\mathrm{Zn})$ is computed instead of $\mathrm{Pb} / \mathrm{Zn}$ because the latter diverges when $\mathrm{Zn}$ concentrations are small.

By itself, Zn had a small weekly cycle with a Sunday minimum. Neither vanadium nor selenium had a statistically significant weekly cycle. The data for copper are noisy.

\subsection{Optical properties of aerosols}

Changes in the optical properties of aerosols at two sites in the central United States are consistent with the weekly cycles from the IMPROVE network. These NOAA sites use completely different instrumentation than the IMPROVE sites and operate continuously rather than sampling for $24 \mathrm{~h}$ every third day. At both NOAA sites, there was a weekly minimum in aerosol light absorption on Monday (Fig. 9). Although the national minimum for IMPROVE elemental carbon was on Sunday, the clusters of sites close to Bondville and Southern Great Plains had minima on Monday (Fig. 3). With samples every third day, there were not enough data to do the direct comparison of just the Bondville IMPROVE site with the NOAA Bondville data. There were no statistically significant weekly cycles in light scattering at either NOAA site. This is consistent with the lack of a statistically significant weekly cycle in sulfate and the small cycle in organic carbon.

\section{Implications}

The weekly cycles shown here are unlikely to be from local sources at the sampling sites for two reasons. First, the data show regional rather than local patterns. Second, with the urban sites excluded many of the remaining IMPROVE sites are at national parks and monuments. One would expect weekend maxima rather than minima if local traffic were generating the weekly cycles.

Large-scale weekly cycles in aerosol have implications for emissions, health effects, and climate studies. The weekly cycles in emissions must be much stronger than the up to $20 \%$ cycles observed in aerosol species. At any site and time, some sources will be very close, some one day upwind, and others further upwind. Multiple sources and variations in winds will both tend to smear out the weekly cycle in emissions.

Weekly cycles of traffic emissions have been studied in California. There, passenger vehicle travel is similar on weekdays and weekends but heavy truck emissions decrease by 70 to $80 \%$ on weekends (Marr et al., 2002; Chinkin et al., 2003; Motallebi et al., 2003; Harley et al., 2005). For passenger vehicles, there are diurnal differences between weekday and weekend. The peak emissions are at rush hours during
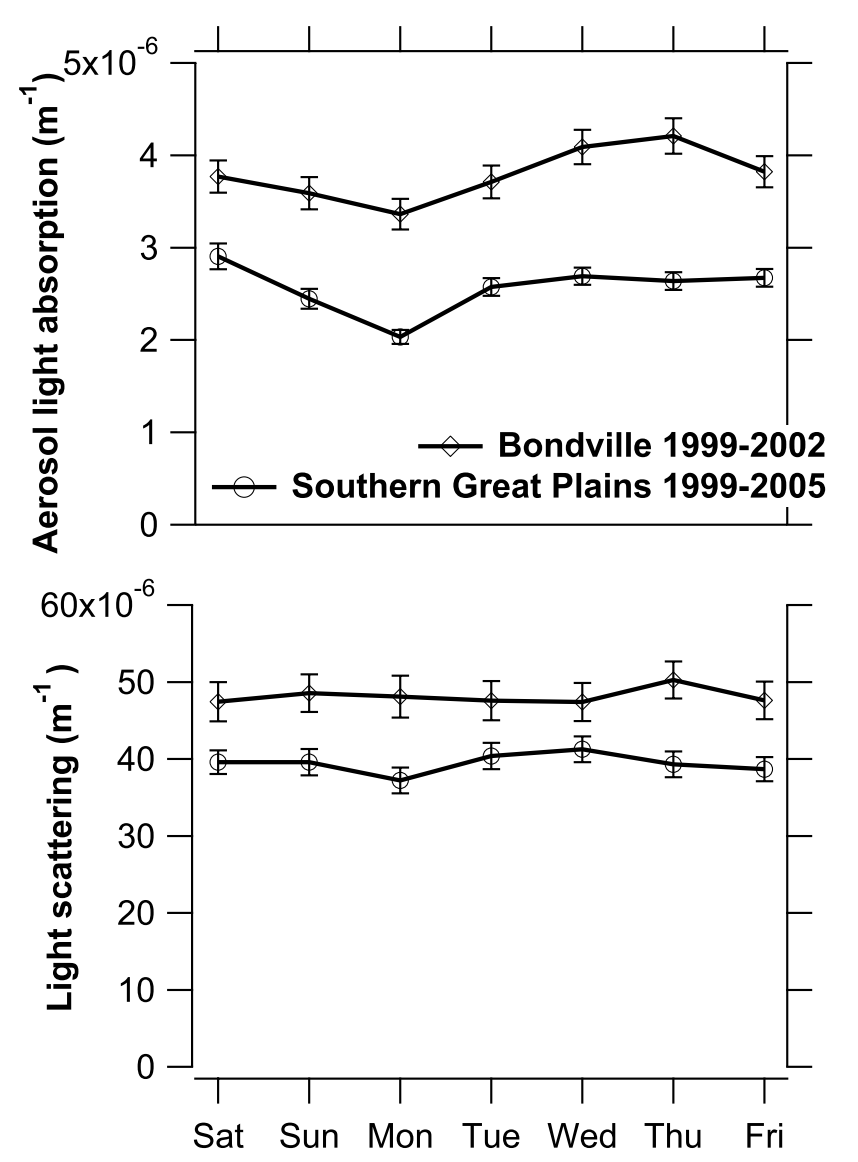

Fig. 9. Aerosol light absorption and scattering from the NOAA monitoring sites at Bondville, Illinois, and Southern Great Plains, Oklahoma.

the week but around midday on weekends. The dominant effect for elemental carbon emissions is the decrease of diesel emissions on weekends. Elemental carbon concentrations on all days should decrease as new controls on heavy truck emissions penetrate the fleet.

The weekly cycle in crustal elements implies a large anthropogenic source in the United States besides wind-blown dust. The crustal elements have an even larger weekly cycle than elemental carbon, which is affected by the $70 \%$ decrease in heavy truck emissions on weekends. That means that the anthropogenic source for crustal elements must be both large and have decrease very significantly on weekends. The larger weekly cycle for crustal elements and PM10 compared to $\mathrm{PM}_{2.5}$ is consistent with the analysis by Bell et al. (2008). Light vehicle traffic on dirt or dusty roads probably does not have a sufficiently large weekly cycle. We do not know what fractions of plowing and other agricultural operations are conducted on weekends, and these may vary with region and season. Construction and heavy truck traffic (Venkatram et al., 1999; Muleski et al., 2005) are possible sources of dust with large weekly cycles. For Pb, the IMPROVE data support a significant source from leaded aviation fuel. 
Weekly cycles may have implications for epidemiological studies of the health effects of aerosols. First, personal exposure to certain species will be affected if people spend more time outdoors on Sundays when mineral dust and elemental carbon concentrations are lower. Second, there might be some effects on the statistics in time series studies. Such studies often include day-of-week in the statistical analysis (Pope and Dockery, 2006). This should take out the biggest effects but it is hard to exclude subtle interactions in the time series. On the other hand, the consistency of weekly cycles throughout the United States means that studies comparing different cities over the same time period should compensate very well for any weekly cycles. Third, health care can be better on weekdays than weekends, resulting in different mortality rates for the same conditions (Bell and Redelmeier, 2001). This could conceivably cause aerosol-health correlations to be underestimated if aerosol health effects maximize on weekdays when treatment is better. Finally, it is possible that the differing weekly cycles of elemental carbon and sulfate could be used in a statistical sense to examine the difficult question of what aerosol components are most responsible for the health effects.

These results are important for studies of weekly and other short-term climate effects of aerosols. The direct climate effects of elemental carbon and non-absorbing fine particle mass have opposite signs. It will not be possible to relate weekly cycles in temperature or precipitation to aerosols without considering elemental carbon separately from other species. The differing weekly cycles of various aerosol species could provide a powerful check on aerosol transport models. The species with the biggest weekly cycles, crustal material and elemental carbon, are insoluble. The most common soluble species is sulfate, which shows no significant weekly cycle. Therefore, weekly cycles in cloud condensation nuclei are likely to be small unless there are systematic changes in the size distribution. Finally, mineral dust particles can be potent ice nuclei so the strong weekly cycle in crustal elements could affect ice clouds and precipitation.

Acknowledgements. S. Capps was supported by the National Oceanic and Atmospheric Administration's Ernest F. Hollings Undergraduate Scholarship Program administered by the Oak Ridge Institute for Science and Education.

Edited by: A. Laaksonen

\section{References}

Almeida, S. M., Pio, C. A., Freitas, M. C., Reis, M. A., and Trancoso, M. A.: Source apportionment of atmospheric urban aerosol based on weekdays/weekends variability: evaluation of road resuspended dust contribution, Atmos. Environ., 40, 2058-2067, 2006.
Anderson, T. L., Charlson, R. J., Winker, D. M., Ogren, J. A., and Holmén, K.: Mesoscale variations of tropospheric aerosols, J. Atmos. Sci., 60, 119-136, 2003.

Bassett, M. E., and Seinfeld, J. H., Atmospheric equilibrium model of sulfate and nitrate aerosols, II. Particle size analysis, Atmos. Environ., 18, 1163-1170, 1984.

Bäumer, D. and Vogel, B.: An unexpected pattern of distinct weekly periodicities in climatological variables in Germany, Geophys. Res. Lett., 34, L03819, doi:10.1029/2006GL028559, 2007.

Bäumer, D., Rinke, R., and Vogel, B.: Weekly periodicities of aerosol optical thickness over Central Europe - evidence of an anthropogenic direct aerosol effect, Atmos. Chem. Phys., 8, 8390, 2008.

Beirle, S., Platt, U., Wenig, M., and Wagner, T.: Weekly cycle of $\mathrm{NO}_{2}$ by GOME measurements: a signature of anthropogenic sources, Atmos. Chem. Phys., 3, 2225-2232, 2003, http://www.atmos-chem-phys.net/3/2225/2003/.

Bell, C. M., and Redelmeier, D. A.: Mortality among patients admitted to hospitals on weekends as compared with weekdays, New England J. Med., 345, 663-668, 2001.

Bell., T. L., Rosenfeld, D., Kim, K.-M., Yoo, J.-M., Lee, M.-I., and Hahnenberger, M., Midweek increase in U.S. summer rain and storm heights suggests air pollution invigorates rainstorms, J. Geophys. Res., 113, D02209, doi: 10.1029/2007JD008623, 2008.

Blanchard, C. L., and Tanenbaum, S. J.: Differences between weekday and weekend air pollutant levels in Southern California, J. Air Waste Manage., 53, 816-828, 2003.

Bond, T. C., Anderson, T. L., and Campbell, D.: Calibration and intercomparison of filter-based measurements of visible light absorption by aerosols, Aerosol Sci. Technol. 30, 582-600, 1999.

Chinkin, L. R., Coe, D. L., Funk, T. H., Hafner, H. R., Roberts, P. T., Ryan, P. A., and Lawson, D. R.: Weekday versus weekend activity patterns for ozone precursors in California's South Coast Air Basin, J. Air Waste Manage., 53, 829-843, 2003.

Chow, J. C., Watson, J. G., Chen, L.-W. A., Chang, M. C. O., Robinson, N. F., Trimble, D., and Kohl, S.: The IMPROVE_A temperature protocol for thermal/optical carbon analysis: maintaining consistency with a long-term database, J. Air Waste Manage., 57, 1014-1023, 2007.

Delene, D. J., and Ogren, J. A.: Variability of aerosol optical properties at four North American surface monitoring sites, J. Atmos. Sci., 59, 1135-1150, 2002.

Elkus, B., and Wilson, K. R.: Photochemical air pollution: Weekend-weekday differences, Atmos. Environ., 11, 509-515, 1977.

Forster, P. M. de F., and Solomon, S.: Observations of a "weekend effect" in diurnal temperature range, Proc. Nat. Acad. Sci. USA, 100, 11225-11230, 2003.

Gong, D.-Y., Guo, D., and Ho, C.-H.: Weekend effect in diurnal temperature range in China: Opposite sign between winter and summer, J. Geophys. Res., 111, D18113, doi:10.1029/2006JD007068, 2006.

Harley, R. A., Marr, L. C., Lehner, J. K., and Giddings, S. N.: Changes in motor vehicle emissions on diurnal to decadal time scales and effects on atmospheric composition, Environ. Sci. Technol., 39, 5356-5362, 2005.

Jin, M., Shepherd, J. M., and King, M. D.: Urban aerosols and their variations with clouds and rainfall: A case study 
for New York and Houston, J. Geophys. Res., 110, D10S20, doi:10.1029/2004JD005081, 2005.

Lough, G. C., Schauer, J. J., and Lawson, D. R.: Day-of-week trends in carbonaceous aerosol composition in the urban atmosphere, Atmos. Environ., 40, 4137-4149, 2006.

Malm, W. C. and Hand, J. L.: An examination of the physical and optical properties of aerosols collected in the IMPROVE program, Atmos. Environ., 41, 3407-3427, 2007.

Malm, W. C., Schichtel, B. A., Pitchford, M. L., Ashbaugh, L. L., and Eldred, R. A.: Spatial and monthly trends in speciated fine particle concentration in the United States, J. Geophys. Res. 109, D03306, doi:10.1029/2003JD003739, 2004.

Marr, L. C., and Harley, R. A.: Modeling the effect of weekdayweekend differences on motor vehicle emissions on photochemical air pollution in central California, Environ. Sci. Technol., 36, 4099-4106, 2002.

Marr, L. C., Black, D. R., and Harley, R. A.: Formation of photochemical air pollution in central California 1. Development of a revised motor vehicle emission inventory, J. Geophys. Res., 107, D64047, doi:10.1029?2001JD000689, 2002.

Millstein, D. E., Harley, R. A., and Hering, S. V.: Weekly cycles in fine particulate nitrate, Atmos. Environ., in press, 2007.

Motallebi, N., Tran, H., Croes, B. E., and Larsen, L. C.: Day-ofweek patterns of particulate matter and its chemical components at selected sites in California, J. Air Waste Manage., 53, 876888, 2003.

Muleski, G. E., Cowherd, C. Jr., and Kinsey, J. S.: Particulate emissions from construction activities, J. Air Waste Manage., 55, 772-783, 2005.

Murphy, D. M., Cziczo, D. J., Froyd, K. D., Hudson, P. K., Matthew, B. M., Middlebrook, A. M., Peltier, R. E., Sullivan, A., Thomson, D. S., and Weber, R. J.: Single-particle mass spectrometry of tropospheric aerosol particles, J. Geophys. Res., 111, D23S32, 2006.

Murphy, D. M., Hudson, P. K., Cziczo, D. J., Gallavardin, S., Froyd, K. D., Johnston, M. V., Middlebrook, A. M., Reinard, M. S., Thomson, D. S., Thornberry, T., and Wexler, A. S.: Distribution of lead in single atmospheric particles, Atmos. Chem. Phys., 7, 3195-3210, 2007,

http://www.atmos-chem-phys.net/7/3195/2007/.
Murphy, J. G., Day, D. A., Cleary, P. A., Wooldridge, P. J., Millet, D. B., Goldstein, A. H., and Cohen, R. C.: The weekend effect within and downwind of Sacramento Part 1: Observations of ozone, nitrogen oxides, and VOC reactivity, Atmos. Chem. Phys., 7, 5327-5339, 2007, http://www.atmos-chem-phys.net/7/5327/2007/.

Novakov, T., Menon, S., Kirchstetter, T. W., Koch, D., and Hansen, J. E.: Aerosol organic carbon to black carbon ratios: Analysis of published data and implications for climate forcing, J. Geophys. Res., 110, D21205, doi: 10.1029/2005JD005977, 2005.

Pope III, C. A. and Dockery, D. W.: Health effects of fine particulate air pollution: lines that connect, J. Air and Waste Manage., 56, 709-724, 2006.

Venkatachari, P., Zhou, L. M., Hopke, P. K., Felton, D., Rattigan, O. V., Schwab, J. J., and Demerjian, K. L.: Spatial and temporal variability of black carbon in New York City, J. Geophys. Res., 111, D10S05, doi:10.1029/2005JD006314, 2006.

Venkatram, A., Fitz, D., Bumiller, K., Du, S., Boeck, M., Ganguly, C.: Using a dispersion model to estimate emission rates of particulate matter from paved roads. Atmos. Environ., 33, 1093-1102, 1999.

Virkkula, A., Ahlquist, N. C., Covert, D. S., Arnott, W. P., Sheridan, P. J., Quinn, P. K., and Coffman, D. J.: Modification, calibration and a field test of an instrument for measuring light absorption by particles, Aerosol Sci. Technol., 39, 68-83, 2005.

White, W. H., Macias, E. S., Miller, D. F., Schorran, D. E., Hoffer, T. E., and Rogers, D. P.: Regional transport of the urban workweek: Methylchloroform cycles in the Nevada-Arizona desert, Geophys. Res. Lett., 17, 1081-1084, 1990.

White, W. H., Macias, E. S., Miller, D. F., Schorran, D. E., Hoffer, T. E., and Rogers, D. P.: Observations of Los Angeles smog in southern Nevada, in: Tropospheric Ozone and the Environment, edited by: R. L. Berglund, Air Waste Management Association, Pittsburgh, 1991. 